\title{
AVALIAÇÃO DO CRESCIMENTO DO LENHO DE Araucaria angustifolia NO PLANALTO NORTE DE SANTA CATARINA
}

\author{
Joanna Rebelo de Oliveira ${ }^{1}$, Eduardo Adenesky-Filho ${ }^{1}$, Karin Esemann-Quadros ${ }^{1,2 *}$ \\ ${ }^{1}$ Universidade Regional de Blumenau, Programa de Pós-Graduação em Engenharia Florestal, Blumenau, Santa Catarina, Brasil - \\ joannarebelo89@gmail.com; eduardo_florestal@hotmail.com \\ ${ }^{2 *}$ Universidade da Região de Joinville, Departamento de Ciências Biológicas, Joinville, Santa Catarina, Brasil - karinesemann@ gmail.com
}

Recebido para publicação: 19/09/2015 - Aceito para publicação: 11/03/2017

\begin{abstract}
Resumo
O presente trabalho apresenta resultados sobre a análise do crescimento de Araucaria angustifolia (Bertol.) O.Kuntze (Araucariaceae) no planalto norte de Santa Catarina, relativos à estimativa da idade, incremento do lenho, cronologias e correlações com temperatura e precipitação. Foram coletadas amostras de madeira de 74 árvores de duas áreas, uma em São Bento do Sul (SB) e outra em Campo Alegre (CA), com auxílio de trado de incremento de $5 \mathrm{~mm}$ de diâmetro, que foram secas ao ar livre e polidas. As camadas de crescimento foram contadas e mensuradas no Image ProPlus, codatados com o auxílio do software COFECHA e as séries temporais foram utilizadas para produzir cronologias no software ARSTAN. A idade das árvores variou de (19) 38 (65) anos em SB e de (34) 62 (123) anos, em CA. O incremento médio foi de $(0,03) 3,86(16,46) \mathrm{mm}$ com desvio padrão de $\pm 2,16 \mathrm{~mm}$ em SB e de $(0,08) 2,56(12,79) \mathrm{mm}$ e desvio padrão de $\pm 1,67 \mathrm{~mm}$ em CA. A correlação com variáveis meteorológicas indicou que o incremento do lenho de A. angustifolia no planalto norte de Santa Catarina é inversamente proporcional à temperatura média no inverno e primavera do ano corrente, antes e durante a fase de crescimento, e diretamente proporcional à precipitação média no verão anterior à fase de crescimento.
\end{abstract}

Palavras-chave: Araucariaceae; camadas de crescimento; idade das árvores; incremento do lenho.

\begin{abstract}
Wood growth analysis of Araucaria angustifolia in north plateau of Santa Catarina. This paper presents results on Araucaria angustifolia (Bertol.) O.Kuntze (Araucariaceae) growth analysis in north plateau of Santa Catarina, related with age of the trees, wood increase, chronologies and correlations with temperature and rainfall. Samples were collected from 74 trees in two areas, in São Bento do Sul (SB) and Campo Alegre (CA), with an increment borer of $5 \mathrm{~mm}$ in diameter. The samples were air dried and polished, the growth rings were counted and measured in Image ProPlus software, crossdated with COFECHA software and the time series were used to produce chronologies in ARSTAN software. The age of trees varied from (19) 38 (65) years in SB and (34) 62 (123) years in CA. The average increase was (0.03) 3.86 (16.46) SD $\pm 2.16 \mathrm{~mm}$ in $\mathrm{SB}$ and (0.08) $2.56(12.79) \mathrm{SD} \pm 1.67 \mathrm{~mm}$ in CA. The correlation with meteorological variables indicated that the wood increasing of A. angustifolia in northern Santa Catarina plateau is inversely proportional to the average temperature in winter and spring of the current year, before and during the growth phase, and directly proportional to the average rainfall in the summer before the growth phase.

Keywords: Araucariaceae; growth rings; tree age; wood increment.
\end{abstract}

\section{INTRODUÇÃO}

Ao longo das últimas décadas, as florestas nativas foram severamente afetadas pelo uso indevido dos recursos florestais. No sul do Brasil, a Floresta Ombrófila Mista (FOM) foi largamente explorada devido à alta qualidade da madeira de várias de suas espécies economicamente importantes, como Araucaria angustifolia (Bertol.) O.Kuntze (Araucariaceae), e sua grande disponibilidade nesta formação florestal.

Considerada como em perigo de extinção (BRASIL, 2014), A. angustifolia ocorre principalmente no Sul e Sudeste do Brasil, Nordeste da Argentina (Missiones) e Sudeste do Paraguai (Alto Paraná). As árvores são perenifólias e chegam a atingir $40 \mathrm{~m}$ de altura e $1,5 \mathrm{~m}$ de diâmetro na fase adulta. O tronco é reto e quase cilíndrico, com fuste podendo alcançar $20 \mathrm{~m}$ ou mais de comprimento e livre de ramos. A copa é umbeliforme nas adultas e piramidal, oval ou colunar nas jovens (WEHR; TOMAZELLO-FILHO, 2000). A casca externa, rugosa, áspera e marrom-arroxeada, se desprende em lâmina na parte superior do caule; a casca interna é resinosa, com tons róseos. A espécie é dióica, isto é, plantas distintas produzem pólen em microestróbilos e sementes em megaestróbilos, raramente sendo encontradas plantas monoicas.

O lenho de A. angutifolia é de cor branco-amarelada, com cerne e alburno pouco diferenciados, textura fina e uniforme, grã direita, superfície lisa ao tato, medianamente lustroso, cheiro e gosto de resina muito pouco intensos. É

FLORESTA, Curitiba, PR, v. 47, n. 2, p. 155 - 164, abr. / jun. 2017.

Oliveira, J. R. de, et al.

ISSN eletrônico 1982-4688

DOI: $10.5380 /$ rf.v47i1.43134 
classificada como leve quanto à densidade - 0,48, 0,53 e $0,50 \mathrm{~g} / \mathrm{cm}^{3}$ para as classes dominante, codominante e dominada, respectivamente (WEHR; TOMAZELLO-FILHO, 2000). Apresenta camadas de crescimento notadas macroscopicamente as quais, segundo Oliveira et al. (2009a; 2009b), são formadas entre a primavera e o outono devido a variações no comprimento do dia e na temperatura, considerados os principais fatores que influenciam na atividade cambial sazonal da araucária nas áreas estudadas pelos autores no Rio Grande do Sul.

O potencial de consumo da madeira faz com que informações sobre sua ecologia e crescimento sejam fundamentais para embasar reflorestamentos, manejos racionais, recomposições e conservação dos remanescentes florestais. Desta forma, o estudo das camadas de crescimento do lenho é uma alternativa para a obtenção de informações acuradas sobre o crescimento das árvores em épocas passadas, dados importantes para descrever o desenvolvimento da espécie.

Sobre a dendrocronologia de A. angustifolia destacam-se os trabalhos de Wehr e Tomazello-Filho (2000), que realizaram a caracterização das camadas de crescimento através da microdensitometria de raios-X; Oliveira et al. (2009a, 2009b), que avaliaram o tempo de formação das camadas de crescimento através de evidências anatômicas e dendrocronológicas e estudaram as relações entre as variações regionais ambientais e a formação intra-anual da madeira em Bom Jesus e Caxias do Sul, no Rio Grande do Sul, e em São Joaquim, em Santa Catarina; Zanon e Finger (2010), que avaliaram a influência dos fatores meteorológicos no crescimento da espécie em floresta plantada em São Francisco de Paula, no Rio Grande do Sul; e Adenesky-Filho (2014), que avaliou a dendroecologia de A. angustifolia na bacia do rio Tibagi, no Paraná, bem como comparou o incremento diametral da espécie em área de encosta e em área de planície.

Poucos são os trabalhos sobre a dendrocronologia e a influência de fatores meteorológicos sobre o crescimento de A. angustifolia em Santa Catarina. Partindo do pressuposto de que o crescimento de araucária no planalto norte de Santa Catarina é influenciado por fatores climáticos, assim como é em outras regiões (OLIVEIRA, 2009a, 2009b; ZANON; FINGER, 2010; CATTANEO et al., 2013), o presente trabalho teve como objetivo avaliar o crescimento da espécie na região de São Bento do Sul e Campo Alegre, em Santa Catarina, incluindo a estimativa da idade, a determinação do incremento do lenho, a construção de cronologias e o estabelecimento de correlações com temperatura e precipitação locais.

\section{MATERIAL E MÉTODOS}

\section{Caracterização das áreas de estudo}

$\mathrm{O}$ trabalho foi realizado em duas áreas de estudo na região norte de Santa Catarina, uma no município

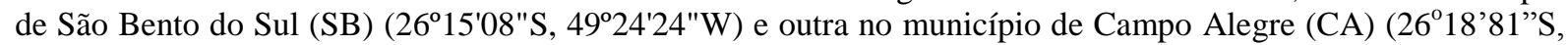
49³6’16”W). Em ambas as áreas a unidade fitogeográfica é Floresta Ombrófila Mista (FOM). Os municípios situam-se em altitude média de 870 m s.n.m, com variação de 800 e 951 m s.n.m. O relevo em CA é plano, enquanto que em SB é mais inclinado.

O clima é do tipo Cfa (clima subtropical úmido), de acordo com a classificação climática de KöppenGeiger (AYOADE, 2007). A distribuição de chuvas é regular no decorrer do ano, com a precipitação média de $116 \mathrm{~mm}$ no mês menos chuvoso (agosto) e $227 \mathrm{~mm}$ no mês mais chuvoso (janeiro). A temperatura média é de $12,3^{\circ} \mathrm{C}$ no mês mais frio (julho) e $20,7^{\circ} \mathrm{C}$ no mês mais quente (fevereiro) (Figura 1 ). Os dados climáticos de precipitação e temperatura foram obtidos da Estação Meteorológica de Rio Negrinho (26¹4' 53"S e 49³4'49"W; altitude: $862 \mathrm{~m}$ ), município vizinho, distando de 17,1 km da área SB e 22,8 km da área CA.

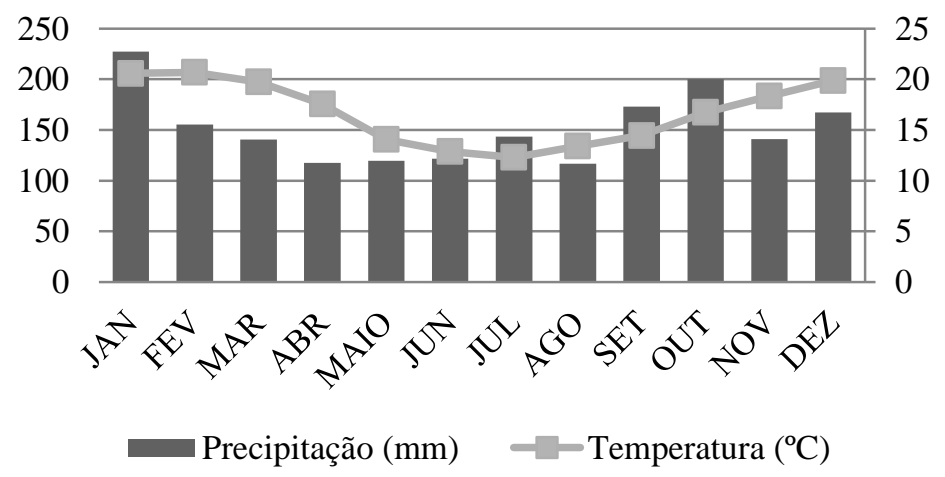

Figura 1. Precipitação total mensal e temperatura média mensal (médias de 1990-2011) na região de Rio Negrinho, SC. Fonte: CIRAM/EPAGRI, Estação Meteorológica de Rio Negrinho, SC.

Figure 1. Total monthly precipitation and average temperatures (1990-2011) in the region of Rio Negrinho, SC. Source: CIRAM / EPAGRI, Meteorological Station Rio Negrinho, SC. 


\section{Coleta do lenho e mensuração das camadas de crescimento}

Foram selecionadas 44 árvores de A. angustifolia em SB (Figura 2a) e 30 árvores em CA (Figura 2b), de forma aleatória, porém optando-se por árvores de fuste reto e íntegro, sem bifurcações ou cicatrizes, distantes mais de 5,0 m de curso d'água. Cada árvore teve seu diâmetro a altura do peito (dap) aferido pela mensuração da circunferência a altura do peito (cap). Amostras de madeira foram tombadas na coleção JOIw com os números de registro CA 1036 e SB 1037.
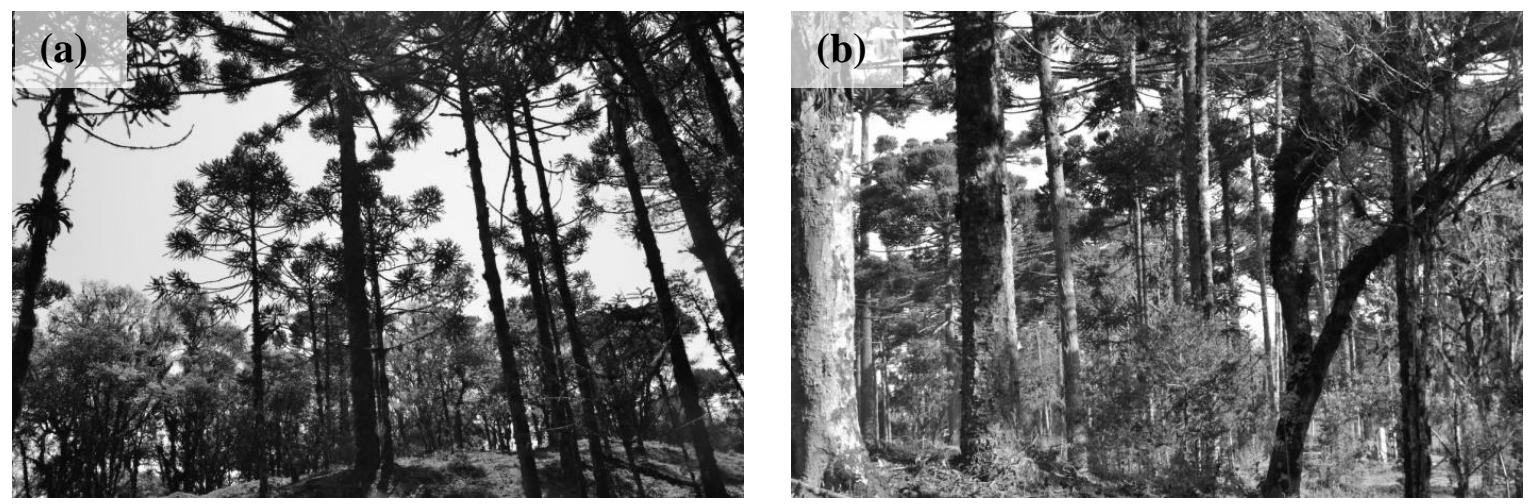

Figura 2. Áreas de amostragem de Araucaria angustifolia em São Bento do Sul (a) e Campo Alegre (b). Figure 2. Araucaria angustifolia sampling areas in São Bento do Sul (a) and Campo Alegre (b).

As coletas de lenho foram realizadas em março de 2012 e entre fevereiro e outubro de 2014. Utilizou-se um trado de incremento com diâmetro de 5,00 mm, retirando-se quatro amostras (baquetas) de cada árvore, perpendiculares entre si e no sentido casca-medula, à altura do peito $(1,30 \mathrm{~m})$. As baquetas foram acondicionadas em canudos plásticos, devidamente identificados, para garantir a integridade das amostras durante o transporte até o Laboratório de Anatomia da Madeira e Dendrocronologia da Universidade Regional de Blumenau (FURB).

No laboratório, as baquetas foram coladas sobre suporte de madeira, com a superfície transversal disposta para cima e expostas ao ar em temperatura ambiente para secagem. Em seguida, foram polidas com papel microabrasivo com granulometria crescente (60 a 600 grãos $/ \mathrm{mm}^{2}$ ) até a obtenção de superfície nítida para exame sob microscópio estereoscópico. As camadas de crescimento foram marcadas a lápis e contadas, permitindo a estimativa da idade das árvores.

Todas as baquetas polidas foram digitalizadas em scanner (resolução 1.200 dpi), acompanhadas de escala. A largura das camadas de crescimento (incremento corrente anual) foi mensurada utilizando-se o software de análise de imagens Image ProPlus, com precisão de 0,001 mm. Os dados foram exportados para Microsoft Office Excel para elaboração de planilhas e gráficos.

\section{Análise de dados}

Para as amostras coletadas em março de 2012, considerou-se ano de formação do último anel completo correspondendo a 2011. As amostras coletadas em fevereiro de 2014 tiveram seu último anel completo formado em 2012, pois o anel que corresponde ao ano de 2013 estava incompleto e não foi incluído. As amostras coletadas entre março e setembro de 2014 tiveram seu último anel completo correspondendo ao ano de 2013.

O controle de qualidade e a verificação da sincronização foram realizados utilizando-se o programa COFECHA (HOLMES et al., 1986). O software foi usado inicialmente para avaliar a sincronia das quatro séries de camadas de crescimento na mesma árvore e, posteriormente, entre árvores. Utilizou-se o Microsoft Office Excel para análise gráfica e sincronização do padrão de crescimento entre as árvores. O programa ARSTAN (COOK; HOLMES, 1984; HOLMES, 2001) foi usado para padronizar todas as séries em uma única série master, removendo as tendências (detrending) e transformando os incrementos correntes em índices de largura de crescimento.

As séries temporais foram sincronizadas e padronizadas, primeiramente, em função de cada área (SB e CA) e, posteriormente, agrupadas, obtendo-se uma cronologia regional (SB + CA). Os intervalos ('janelas') utilizados foram de 20 anos, o que garantiu o melhor ajuste com os dados, já que algumas árvores são jovens, como indicado na tabela 1. A qualidade da cronologia foi avaliada pelo valor de SEP (sinal expresso da população), cujos valores forneceram informações que confirmaram a existência de sinais comuns entre as árvores, bem como a qualidade da avaliação das respostas do clima ao crescimento (MÉRIAN et al., 2013).

Para avaliar a existência de diferenças entre os incrementos anuais das árvores localizadas em São Bento do Sul e Campo Alegre, foi realizada a comparação de medianas pelo teste não paramétrico Kruskal-

FLORESTA, Curitiba, PR, v. 47, n. 2, p. 155 - 164, abr. / jun. 2017.

Oliveira, J. R. de, et al.

ISSN eletrônico 1982-4688

DOI: $10.5380 /$ rf.v47i1.43134 
Wallis (BRIENEN; ZUIDEMA, 2006), evitando violar o pressuposto de independência entre as observações. O resultado diferenciado entre as medianas foi apresentado na forma de gráfico do tipo Box Plot. As análises estatísticas foram efetuadas com auxílio do software PAST versão 3.1 (HAMMER et al., 2001).

Para averiguar as relações entre o crescimento das árvores e as variáveis meteorológicas mensais, as cronologias foram correlacionadas (correlação de Pearson) com as séries históricas de precipitação total e temperatura média mensais, obtidas da estação meteorológica da CIRAM/EPAGRI em Rio Negrinho, SC. As cronologias foram analisadas separadamente para cada área de coleta (SB e CA) e, posteriormente, para a região $(\mathrm{SB}+\mathrm{CA})$.

As séries de crescimento foram correlacionadas com cada uma das variáveis meteorológicas mensais, combinando as séries para o mesmo ano ou para o ano anterior. Para o arranjo resultante das correlações entre variáveis, foi gerado um perfil gráfico de correlações (correlação de Pearson), ressaltando os períodos de influência positiva ou negativa dos fatores meteorológicos no crescimento das árvores. As análises foram estabelecidas em planilhas do Microsoft Office Excel.

\section{RESULTADOS}

\section{Estimativa da idade e determinação do incremento}

Através da contagem e mensuração da largura das camadas de crescimento, todas as árvores tiveram a idade determinada e seu incremento anual estabelecido. A árvore mais jovem, com 19 camadas de crescimento, foi coletada em São Bento do Sul (SB) e a mais velha, com 123 camadas, foi coletada em Campo Alegre (CA). As árvores amostradas em SB apresentaram um incremento radial médio de $3,86 \mathrm{~mm}$, valor mais alto em comparação aos 2,56 mm das árvores em CA. Além do incremento radial médio superior, as árvores de SB apresentaram o menor $(0,03 \mathrm{~mm})$ e o maior $(16,46 \mathrm{~mm})$ incremento radial (Tabela 1$)$.

Tabela 1. Número de árvores amostradas por ano da coleta, área, idade estimada (anos) e incremento radial (milímetros). SB: São Bento do Sul. CA: Campo Alegre. Valores de idade e incremento apresentados na forma de mínimo-média-máximo; entre parênteses está o desvio padrão.

Table 1. Number of trees sampled per year, estimated age (years) and radial increment (millimeters). SB: São Bento do Sul. CA: Campo Alegre. Age values and increase presented in the form minimum-mediummaximum; standard deviation between parenthesis.

\begin{tabular}{lcccc}
\hline Ano de coleta & Área & $\mathbf{N}^{\mathbf{0}}$ de árvores & Idade & Incremento \\
\hline $2012 / 2014$ & SB & 44 & $19-38-65$ & $0,03-3,86-16,46( \pm 2,16)$ \\
2014 & CA & 30 & $34-62-123$ & $0,08-2,56-12,79( \pm 1,67)$ \\
\hline
\end{tabular}

A análise das medianas (Kruskal-Wallis) demonstrou diferença significativa $(\mathrm{p}<0,0001)$ entre os incrementos radiais de A. angustifolia das duas áreas (Figura 3) para todo o período em comum analisado, compreendido entre os anos de 1954 e 2013. O desempenho diferenciado também foi evidenciado quando analisadas (Kruskal-Wallis) as taxas de incremento em intervalos de 10 anos, apresentando diferenças significativas $(\mathrm{p}<0,0001)$ desde as décadas de 1960 até 2010, nas quais as árvores de SB apresentaram maiores valores de incremento.

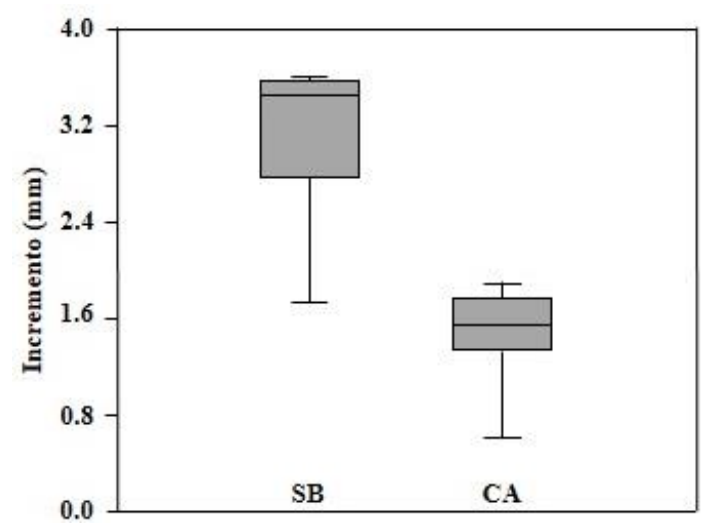

Figura 3. Box plot dos valores de incremento mediando ( $\mathrm{mm})$ de Araucaria angustifolia de São Bento do Sul (SB) e Campo Alegre (CA).

Figure 3. Box plot of median increment values (mm) of Araucaria angustifolia in São Bento do Sul (SB) and Campo Alegre (CA). 


\section{Sincronização e obtenção das séries das camadas de crescimento}

Em SB, das 44 árvores de $A$. angustifolia amostradas, 33 foram satisfatoriamente codatadas; em CA, das 30 árvores coletadas, foram 17. A sincronização a partir do total de 50 árvores de ambas as áreas (SB + CA) resultou em uma intercorrelação de 0,306 e sensibilidade média de 0,316, com uma correlação crítica de 0,5155 para intervalos ('janelas') de 20 anos (Tabela 2).

Tabela 2. Estatística descritiva das codatações das camadas de crescimento de Araucaria angustifolia, nas áreas de São Bento do Sul (SB) e de Campo Alegre (CA) e regional (SB + CA).

Table 2. Descriptive statistics of tree rings crossdating of Araucaria angustifolia in the areas of São Bento do $\mathrm{Sul}(\mathrm{SB})$ and Campo Alegre $(\mathrm{CA})$ and regional ( $\mathrm{SB}+\mathrm{CA})$.

\begin{tabular}{llll}
\hline & SB & CA & SB + CA \\
\hline Período considerado (anos) & $1954-2013$ & $1887-2013$ & $1887-2013$ \\
Número de árvores & $33(44)$ & $17(30)$ & $50(74)$ \\
Número de séries & 78 & 35 & 109 \\
Número de camadas de crescimento & 2690 & 2233 & 5113 \\
Comprimento médio das séries (anos) & 34 & 64 & 47 \\
Intercorrelação das séries & 0,390 & 0,314 & 0,306 \\
Correlação crítica ('janelas' de 20 anos) & 0,5155 & 0,5155 & 0,5155 \\
Sensibilidade média & 0,282 & 0,356 & 0,316 \\
Sinal expresso da população & 0,733 & 0,693 & 0,611 \\
\hline
\end{tabular}

Deformidades anatômicas da madeira, principalmente camadas falsas ou ausentes, flutuações de densidade e deposição de resina dificultaram a codatação, porém não impediram a sincronização. As deformidades foram recorrentes em árvores velhas quando comparadas com árvores novas, especialmente em CA.

\section{Cronologias e relações com variáveis meteorológicas}

A representação gráfica das séries temporais, da cronologia padronizada e do número de árvores utilizadas na sua construção é apresentada na figura 4.

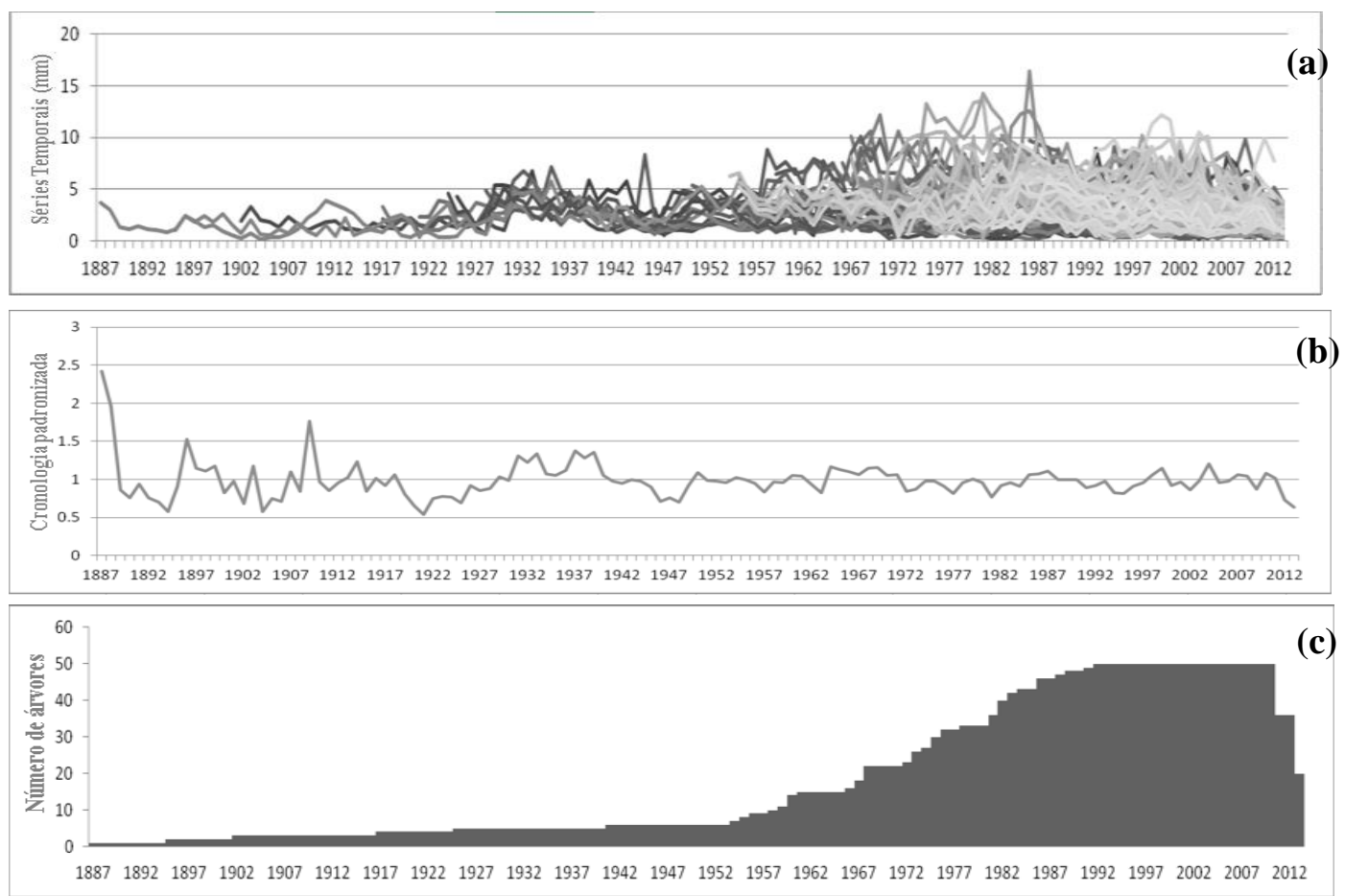

Figura 4. Representação gráfica da sincronização das camadas de crescimento de Araucaria angustifolia regional do planalto norte de Santa Catarina $(\mathrm{SB}+\mathrm{CA})$, com as séries temporais em $\mathrm{mm}(\mathrm{a})$, cronologia padronizada (b) e número de árvores utilizadas por período (c).

Figure 4. Graphic representation of synchronization of Araucaria angustifolia tree rings of northern highlands of Santa Catarina ( $\mathrm{SB}+\mathrm{CA})$, with time series in $\mathrm{mm}(\mathrm{a})$, standard chronology (b) and number of trees used for period (c).

FLORESTA, Curitiba, PR, v. 47, n. 2, p. 155 - 164, abr. / jun. 2017.

Oliveira, J. R. de, et al.

ISSN eletrônico 1982-4688

DOI: $10.5380 /$ rf.v47i1.43134 
Para a identificação das correlações com as variáveis meteorológicas, as cronologias foram analisadas separadamente para cada área de coleta (SB e CA) e, posteriormente, para a região ( $\mathrm{SB}+\mathrm{CA}$ ), considerando os dados de temperatura média e precipitação total mensais.

A cronologia de SB se correlacionou negativamente com a temperatura média mensal no mês de maio precedente e nos meses de novembro e dezembro correntes (Figura 5a). Com a precipitação, verificou-se uma correlação positiva no mês de dezembro precedente e negativa no mês de março corrente (Figura 5b). A cronologia de CA teve correlação positiva com a temperatura no mês de agosto precedente e negativa nos meses de outubro e abril do ano corrente (Figura 5c), não apresentando correlação significativa em nenhum período considerado com a precipitação (Figura $5 \mathrm{~d}$ ). A cronologia regional $(\mathrm{SB}+\mathrm{CA})$ teve correlação negativa com a temperatura nos meses de maio precedente e novembro e dezembro correntes (Figura 5e) e com a precipitação a correlação foi positiva nos meses de dezembro precedente e outubro corrente (Figura 5f).
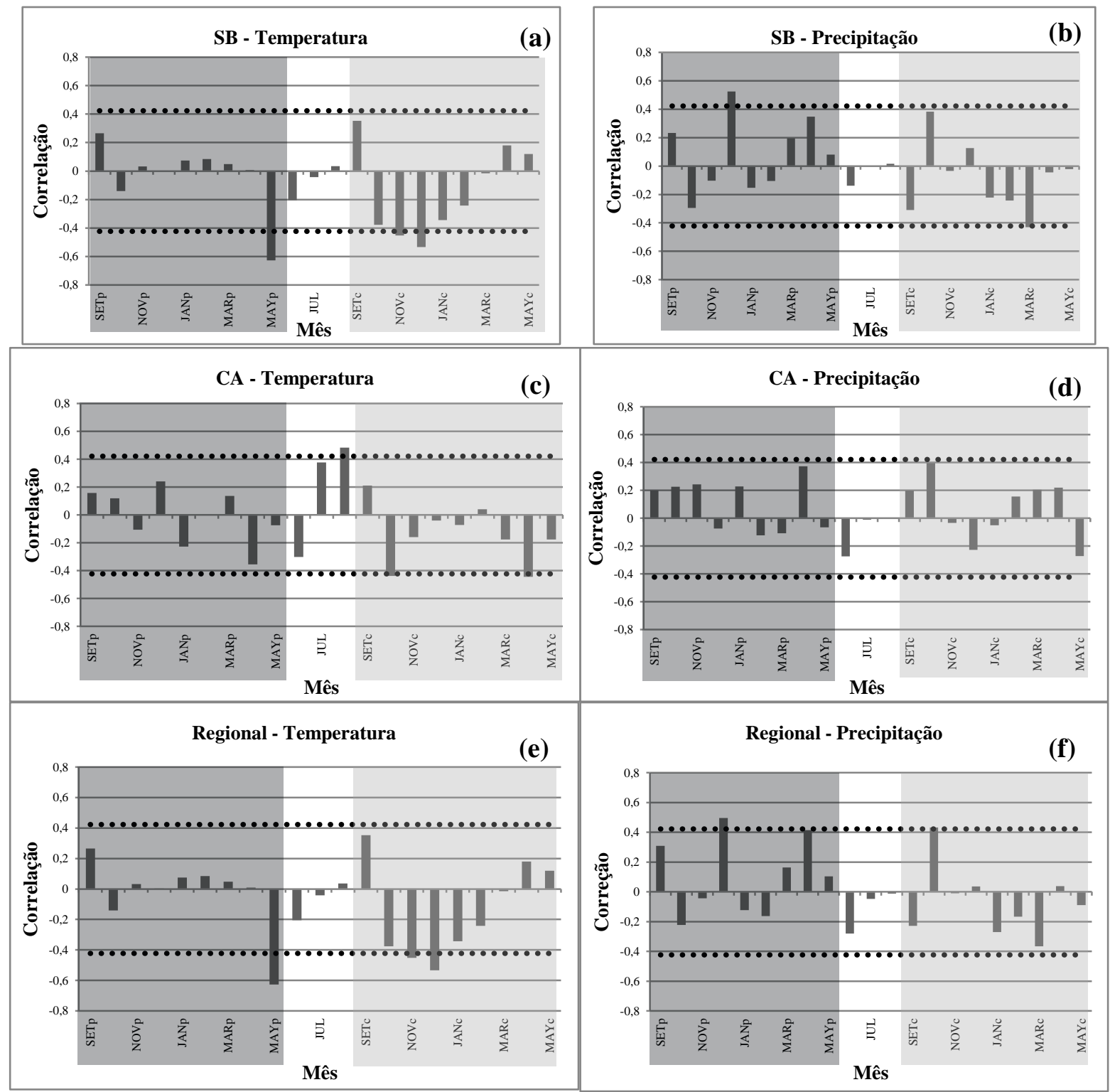

Figura 5. Representação gráfica da correlação das cronologias de Araucaria angustifolia de São Bento do Sul (SB), Campo Alegre (CA) e Regional (SB + CA) com a temperatura média mensal (a, c, e) e com a precipitação total mensal $(b, d, f)$. p: meses do período de crescimento precedente; $c$ : meses do período de crescimento corrente; linha pontilhada: limite de $95 \%$ de confiança.

Figure 5. Correlation of Araucaria angustifolia chronologies of São Bento do Sul (SB), Campo Alegre (CA) and regional area $(\mathrm{SB}+\mathrm{CA})$ in the northern plateau of Santa Catarina with the monthly temperature average $(\mathrm{a}, \mathrm{c}, \mathrm{e})$ and the monthly total rainfall $(\mathrm{b}, \mathrm{d}, \mathrm{f}) . \mathrm{p}$ : months of previous growing period; $\mathrm{c}$ : months of growing season; dotted line: $95 \%$ confidence limit. 


\section{DISCUSSÃO}

\section{Idade e incremento das árvores}

A idade média das árvores de Araucaria angustifolia é de 38 anos em SB e de 62 anos em CA (Tabela 1). As áreas amostradas estão localizadas próximas a núcleos urbanos, o que pode ter influenciado o crescimento das árvores nestas áreas. Em trabalho realizado por Adenesky-Filho (2014) na Bacia do rio Tibagi, no Paraná, as araucárias analisadas apresentavam idade média de 140 e 72 anos em encosta e planície, respectivamente. A população de araucárias pode ter sofrido distúrbios pretéritos, como a exploração madeireira e outras formas de pressão antrópica, estando agora em fase de regeneração na região, situação comprovada nas unidades amostrais em São Bento do Sul e Campo Alegre do Inventário Florístico Florestal de Santa Catarina (VIBRANS et al., 2013). Estas unidades estão inseridas na unidade fitogeográfica Floresta Ombrófila Mista e, segundo dados do Inventário (MEYER et al., 2013), apresentam fisionomia de vegetação florestal secundária em estádio avançado e médio de regeneração. Os resultados fazem supor que a área SB deve ter se regenerado após exploração madeireira há cerca de 50/60 anos sendo, a partir de então, preservada pelos seus proprietários. A área CA, por apresentar árvores mais velhas, com média de idade superior às de $\mathrm{SB}$, passou pelo processo exploratório há mais tempo.

Entretanto, a média de idade observada neste estudo difere do resultado encontrado por Oliveira et al. (2009b) em duas áreas no Rio Grande do Sul. Os autores observaram araucárias com idade média de 122 anos na FLONA de São Francisco de Paula e de 90 anos na Fazenda Monte Negro, que foi fundada há 127 anos e tem sido preservada pelos proprietários desde então. Por se tratar de uma FLONA e de uma propriedade particular, a floresta está preservada, o que justifica serem árvores de idade avançada.

Além disso, em SB e CA ocorrem mais árvores com idade entre 10 e 60 anos e menos com idade superior. As árvores mais velhas ainda eram jovens, com menor diâmetro, na época áurea da exploração madeireira na região, a partir de 1930, tendo escapado do corte. Esta exploração durou até 1960-70, quando foram criadas leis de combate ao desmatamento, aumentando o controle da exploração de determinadas espécies e a fiscalização de serrarias (CABRAL; CESCO, 2008). Como estas árvores atualmente vivem em áreas livres de manejo, as análises dendrocronológicas têm comprovado sua importância no aumento do conhecimento sobre os padrões de crescimento das espécies tropicais, revelando trajetórias de crescimento (ROZENDAL; ZUIDEMA, 2011; COSTA et al., 2015).

As taxas de incremento radial das árvores em SB apresentaram os maiores valores mínimo, médio e máximo em comparação com as de CA, conforme mostrado na tabela 1. Segundo Schweingruber et al. (2006), quanto mais velha for a árvore, menor será a largura da camada de crescimento, ou seja, o incremento do lenho diminui com o aumento da idade da árvore, o que explica a maior média de largura das camadas de crescimento em SB, onde as árvores são mais jovens do que em CA. Entretanto, uma oscilação no crescimento é esperada, podendo ocorrer maior crescimento em consequência de anos com condições mais favoráveis e vice-versa. Mattos et al. (2010) observaram esta oscilação, registrando décadas com aumento no incremento médio anual em nove árvores de A. angustifolia na Reserva Florestal Embrapa/Epagri, em Caçador, Santa Catarina, ao longo de 89 anos. Porém, sua análise foi feita por classe diamétrica (0-20 até mais de $100 \mathrm{~cm}$ de diâmetro) e não por idade das árvores e os resultados obtidos não apresentaram diferenças estatisticamente significativas entre todas as classes analisadas. Segundo os autores, o incremento em diâmetro em árvores adultas tende a estabilizar o longo do tempo, antes de atingir o declínio natural, mas cada árvore ou espécie reflete suas próprias condições e limitações de crescimento. Assim, nem sempre a árvore mais velha é a de maior diâmetro.

Além da idade, outro fator que pode ter influenciado na diferença de incremento observada entre as duas áreas amostradas são as características geopedológicas e geomorfológicas. Na área SB, com relevo mais inclinado, as árvores apresentam maior incremento médio. Da mesma forma, Adenesky-Filho (2014) encontrou araucárias com incremento médio maior em encosta do que em áreas de planície; a presença de diques de diabásio pode ter contribuído para tal resultado. Variações na topografia podem causar diferentes padrões de desenvolvimento e abundância de espécies arbóreas, em escala local, especialmente em função das modificações na fertilidade e profundidade dos solos, drenagem e profundidade do lençol freático e/ou quantidade de serapilheira (BUDKE et al., 2007; GIEHL et al., 2007).

As diferenças no incremento foram evidenciadas pelo teste de Kruskal-Wallis $(\mathrm{p}<0,0001)$ para intervalos de 10 anos. As árvores em SB apresentaram taxas de incremento maiores nas décadas de 1960 até 2010 em relação às de CA. Além disso, as árvores de SB apresentam uma distribuição assimétrica negativa e as de CA uma distribuição simétrica, considerando suas medianas pelo teste aplicado, como mostrado na Figura 3. Da mesma forma, as árvores amostradas por Adenesky-Filho (2014), em encosta também apresentaram maior incremento do que as árvores de planície nas décadas de 1990 e 2000.

FLORESTA, Curitiba, PR, v. 47, n. 2, p. 155 - 164, abr. / jun. 2017.

Oliveira, J. R. de, et al.

ISSN eletrônico 1982-4688

DOI: $10.5380 /$ rf.v47i1.43134 


\section{Séries temporais e padrões de crescimento}

$\mathrm{Na}$ área $\mathrm{CA}$ a intercorrelação entre as árvores foi menor do que em SB, pois a faixa etária das árvores é mais elevada, consequentemente, com características anatômicas peculiares (anéis estreitos, casados, flutuações intra-anuais de densidade, presença de resina, entre outros). Da mesma forma, Oliveira et al. (2009b) no Rio Grande do Sul, Cattaneo et al. (2013) na Argentina e Adenesky-Filho (2014) no Paraná encontraram estes tipos de característica anatômica com o aumento da idade das árvores de A. angustifolia.

Para o conjunto de árvores analisadas, o valor da correlação crítica é de 0,5155 (COFECHA) para codatação significativa à significância de $99 \%$. Embora este valor não tenha sido atingido, os resultados de sincronismo para A. angustifolia foram satisfatórios e não se distanciam de outras cronologias da mesma espécie em diferentes regiões do Brasil e da Argentina (Tabela 3).

Tabela 3. Intercorrelações (r) obtidas para Araucaria angustifolia em estudos dendrocronológicos conduzidos em diferentes regiões do sul do Brasil e na Argentina. UF: Unidade fitogeográfica; FOM: Floresta Ombrófila Mista; FESD: Floresta Estacional Semidecidual. Local: AR - Argentina; PR - Paraná; RS Rio Grande do Sul; SC - Santa Catarina.

Table 3. Intercorrelations (r) obtained for Araucaria angustifolia in dendrochronological studies conducted in different regions of southern Brazil and Argentina. UF: Fitogeographycal Unit; FOM: Rain Forest Mixed; FESD: Semidecidual Seasonal Forest. Location: AR - Argentina; PR - Paraná; RS - Rio Grande do Sul; SC - Santa Catarina.

\begin{tabular}{lrrlc}
\hline Estudos & r & Período considerado & UF & Local \\
\hline Este estudo: Regional & 0,306 & 126 anos $(1887-2013)$ & FOM & SC \\
Este estudo: SB & 0,390 & 59 anos $(1954-2013)$ & FOM & SC \\
Este estudo: CA & 0,314 & 126 anos $(1887-2013)$ & FOM & SC \\
Oliveira et al. (2009b) & 0,250 & 122 anos $(1882-2003)$ & FOM & RS \\
& 0,340 & 143 anos $(1861-2003)$ & Campo & RS \\
Adenesky-Filho (2014)-Regional & 0,318 & 236 anos $(1775-2010)$ & FESD/FOM & PR \\
Adenesky-Filho (2014)-Encosta & 0,401 & 370 anos $(1641-2010)$ & FESD/FOM & PR \\
Adenesky-Filho (2014)-Planície & 0,431 & 236 anos $(1775-2010)$ & FESD/FOM & PR \\
Cattaneo et al. (2013) & 0,460 & 59 anos $(1950-2008)$ & Plantio & AR \\
& 0,510 & 57 anos $(1952-2008)$ & Plantio & AR \\
\hline
\end{tabular}

Fonte: adaptado de Adenesky-Filho (2014).

\section{Camadas de crescimento e fatores meteorológicos}

A correlação obtida indica que o aumento na temperatura média durante os períodos analisados provocou um menor incremento nas árvores de A. angustifolia em SB e CA. Oliveira et al. (2009b) também verificaram que o aumento da temperatura média na estação outonal prévia à estação de crescimento e durante a estação de crescimento provocou um menor crescimento das árvores. Oliveira et al. (2009a) descreveram a atividade cambial sazonal em árvores de A. angustifolia verificando que, durante os meses de maio e junho, outono prévio à estação de crescimento, ocorre o estoque de carboidratos para a formação de madeira. Assim, o aumento da temperatura durante esse período acarreta em diminuição do armazenamento de carboidratos e, consequentemente, redução no crescimento da árvore. Esses resultados sugerem que o aumento da temperatura média ao fím do inverno e na primavera, período anterior e durante a estação de crescimento, afeta negativamente no incremento do lenho das araucárias (SANTAROSA et al., 2007). Portanto, se as mudanças climáticas continuarem no sentido de a temperatura média do planeta aumentar, é de se supor que as árvores de A. angustifolia possam apresentar incremento médio cada vez menor no futuro.

Segundo Molion (2010), as variações climáticas correlacionadas às alterações da temperatura no Pacífico podem estar influenciando na temperatura do ar e nos níveis de precipitação, provocando consequencias graves aos ecossistemas. Plantas submetidas a taxas de temperatura mais elevadas apresentam gastos energéticos superiores para a execução de processos fisiológicos, incluindo os mecanismos de trocas gasosas; os estômatos tendem a fechar para evitar a perda de água e, consequentemente, prejudicando o seu crescimento. Porém, é preciso considerar que não é apenas a variação de temperatura que interfere no crescimento das árvores, mas também outros fatores como a precipitação.

A correlação entre a cronologia regional $(\mathrm{SB}+\mathrm{CA})$ e a precipitação é significativamente positiva para o mês de dezembro precedente ao período de crescimento corrente. Isso mostra que o aumento de chuvas no verão prévio à estação de crescimento acarreta um aumento no incremento diamétrico das árvores, que utilizam o aporte hídrico e temperaturas mais elevadas nos meses iniciais da estação de crescimento para produção de biomassa, refletida na largura das camadas de crescimento. Zanon e Finger (2010) verificaram o crescimento de 
árvores de A. angustifolia ao longo de três anos na FLONA de São Francisco de Paula, no Rio Grande do Sul, identificando que o aumento de temperatura e da precipitação influencia positivamente no crescimento de árvores desta espécie. Apesar de terem trabalhado com uma população de Cedrela fissilis na Floresta Ombrófila Mista do sul do Brasil, Andreacci et al. (2014) também concluíram que a água no início da estação de crescimento é fundamental para que as árvores, no período de produção de novas folhas e flores, tenham maiores condições de aumento de biomassa.

Embora algumas características não tenham sido averiguadas nas áreas estudadas, como diferenças na fertilidade do solo ou as alterações biológicas sofridas pela árvore desde o seu nascimento até o final de seu desenvolvimento, estes fatores podem provocar mudanças de crescimento e influenciar as respostas das árvores a um fator meteorológico comum (SANTAROSA et al., 2007; ADENESKY-FILHO, 2014). Além disso, tendo em vista que os dados meteorológicos utilizados para estas correlações partem de uma mesma estação, as variações microclimáticas podem ser outros fatores causadores de diferenças entre as duas áreas estudadas (SANTAROSA et al., 2007).

\section{CONCLUSÕES}

- A determinação da idade das árvores de Araucaria angustifolia em CA e SB apontou para uma comunidade jovem em fase de regeneração, o que pode estar relacionado a distúrbios naturais ou antrópicos pretéritos.

- O incremento do lenho foi maior nas árvores mais jovens. Além da idade, outros fatores podem contribuir com a diferença de incremento entre árvores de áreas diferentes, como geologia, geomorfologia e topografia, que merecem ser investigados.

- A correlação com variáveis meteorológicas indicou que o incremento do lenho de A. angustifolia no planalto norte de Santa Catarina é inversamente proporcional à temperatura média no inverno e primavera do ano corrente, antes e durante a fase de crescimento, e diretamente proporcional à precipitação média no verão anterior à fase de crescimento.

\section{AGRADECIMENTOS}

Os autores agradecem ao Eng. Florestal Eder de Lima, pela colaboração nos trabalhos em campo, e à CAPES, pela concessão da bolsa de Mestrado à primeira autora.

\section{REFERÊNCIAS}

ADENESKY-FILHO, E. Florística, fitossociologia e dendroecologia em encosta e planície do médio Rio Tibagi, Telêmaco Borba, Paraná, Brasil. 140 f. Tese (Doutorado em Engenharia Florestal) - Setor de Ciências Agrárias, Universidade Federal do Paraná, Curitiba, 2014.

ANDREACCI, F.; BOTOSSO, P. C.; GALVÃO, F. Sinais climáticos em anéis de crescimento de Cedrela fissilis em diferentes tipologias de florestas ombrófilas do Sul do Brasil. Floresta, Curitiba, v. 44, n. 2, p. 323-332, 2014.

AYOADE, J. O. Introdução à climatologia para os trópicos. Rio de Janeiro: Bertrand Brasil, 12 ed. 2007.

BRASIL. Ministério do Meio Ambiente. Portaria MMA nº 443, de 17 de dezembro de 2014, dispõem sobre as espécies da flora brasileira ameaçadas de extinção. Disponível em: http://cncflora.jbrj.gov.br/portal/static/pdf/ portaria_mma_443_2014.pdf >. Acesso em: 17/08/2016.

BRIENEN, R. J. W.; ZUIDEMA, P. A. The use of tree rings in tropical forest management: Projecting timer yields of four Bolivian tree species. Forest Ecology and Management, v. 226, n. 1, p. 256-267, 2006.

BUDKE, J. C.; JARENKOW, J. A.; OLIVEIRA-FILHO, A. T. Relationships between tree component structure, topography and soils of a riverside forest, Rio Botucaraí, Southern Brazil. Plant Ecology, n. 189, p. 187-200, 2007.

CABRAL, D. C.; CESCO, S. Notas para uma história da exploração madeireira na mata atlântica do sul-sudeste. Ambiente e Sociedade, v. XI, n. 1, p. 3-48, 2008.

CATTANEO, N.; PAHR, N.; FASSOLA, H.; LEPORATI, J.; BOGINO, S. Sex-related, growth-climate association of Araucaria angustifolia in the neotropical ombrophilous woodlands of Argentina. Dendrochronologia, n. 31, p. 147-152, 2013.

COOK, E. R.; HOLMES, R. L. User's manual for program ARSTAN. Palisades: Lamont-Doherty Earth Observatory, 1984. 
COSTA, M. S.; FERREIRA, K. E. B.; BOTOSSO, P. C.; CALlADO, C. H. Growth analysis of five Leguminosae native tree species from a seasonal semidecidual lowland forest in Brazil. Dendrochronologia, 36 : 23-32, 2015.

GIEHL, E. L. H.; BUDKE, J. C.; ATHAYDE, E. A. Distribuição espacial de espécies arbóreas em uma Floresta Estacional em Santa Maria, Sul do Brasil. Pesquisas Botânicas, n. 58, p. 215-226, 2007.

HAMMER, O.; HARPER, D. A. T.; RYAN, P. D. 2001. PAST - Palaeontological statistics. Disponível em: http://folk.uio.no/ohammer/past/ $\geq$ Acesso em: 02 de Ago. 2016.

HOLMES, R. L. Dendrochronology program library. Available from the Laboratory of Tree-Ring Research, University of Arizona, Tucson, 2001.

HOLMES, R. L.; ADAMS, R. K.; FRITTS, H. C. Quality control of crossdating and measuring: a user's manual for program COFECHA. In: Tree-ring chronologies of Western North America: California, eastern Oregon and northern Great Basin. Tucson: Arizona University, 1986.

MATTOS, P. P.; OLIVEIRA, M. F.; AGUSTINI, A. F.; BRAZ, E. M.; RIVERA, H.; OLIVEIRA, Y. M. M. ROSOT, M. A. D.; GARRASTAZU, M. C. Aceleração do crescimento em diâmetro de espécies da Floresta Ombrófila Mista nos últimos 90 anos. Pesquisa Florestal Brasileira, v. 30, n. 64, p. 319-326, 2010.

MÉRIAN, P.; PIERRAT, J. C.; LEBOURGEOIS, F. Effect of sampling effort on the regional chronology statistics and climate-growth relationships estimation. Dendrochronologia, 31: 58-67, 2013.

MEYER, L.; SEVEGNANI, L.; GASPER, A. L. de; SCHORN, L. A.; VIBRANS, A. C.; LINGNER, D. V.; SOBRAL, M. G.; KLEMZ, G.; SCHMDT, R.; ANASTÁCIO JUNIOR, C.; BRONGI, E. Fitossociologia do componente arbóreo/arbustivo da Floresta Ombrófila Mista em Santa Catarina. In: VIBRANS, A. C.; SEVEGNANI, L.; GASPER, A. L. de; LINGNER, D. V. (ed.). Inventário Florístico Florestal de Santa Catarina, v. III, Floresta Ombrófila Mista. Blumenau: EDIFURB, p. 157-189, 2013.

MOLION, L. C. B. Variabilidade e alterações climáticas. In: FIGUEIREDO, T.; RIBEIRO, L. F.; RIBEIRO, A. C.; FERNANDES, L. F. (org.). Clima e recursos naturais. Instituto Politécnico de Bragança, Bragança, Portugal, p. 17-41, 2010.

OliveIRA, J. M.; SANTAROSA, E.; PILlAR, V. D.; ROIG, A. R. Seasonal cambium activity in the subtropical rain forest tree Araucaria angustifolia. Trees, n. 23, p. 107-115, 2009a.

OLIVEIRA, J. M.; ROIG, F. A.; PILLAR, V. D. Climatic signals in tree-rings of Araucaria angustifolia in the southern Brazilian highlands. Austral Ecology. p. 1-15, $2009 \mathrm{~b}$.

ROZENDAL, D. M. A.; ZUIDEMA, P. A. Dendroecology in the tropics: a review. Trees, 25(1): 3-16, 2011.

SANTAROSA, E.; OlIVEIRA, J. M.; ROIG, F. A.; PILlAR, V. D. Crescimento Sazonal em Araucaria angustifolia: evidências anatômicas. Revista Brasileira de Biociências, Porto Alegre, v. 5, n. 1, p. 618-620, 2007.

SCHWEINGRUBER, F. H.; BÖRNER, A.; SCHULZE, E.-D. Atlas of woody plant stems: evolution, structure, and environmental modifications. Alemanha: Springer, 2006.

VIBRANS, A. C.; McROBERTS, R. E.; LINGNER, D. V.; NICOLETTI, A. L.; MOSER, P. Extensão original e remanescentes da Floresta Ombrófila Mista em Santa Catarina. In: VIBRANS, A. C.; SEVEGNANI, L.; GASPER, A. L. de; LINGNER, D. V. (ed.). Inventário Florístico Florestal de Santa Catarina, v. III. Floresta Ombrófila Mista. Blumenau: EDIFURB, p. 25-31, 2013.

WEHR, N. J.; TOMAZELLO FILHO, M. Caracterização dos anéis de crescimento de árvores de Araucaria angustifolia (Bert.) O. Ktze, através da microdensitometria de Raio X. Scientia Forestalis, 58, 161-170, 2000.

ZANON, M. L. B.; FINGER, C. A. G. Relação de variáveis meteorológicas com o crescimento das árvores de Araucaria angustifolia (Bertol.) Kuntze em povoamentos implantados. Ciência Florestal, Santa Maria, v. 20, n. 3, p. 467-476, 2010. 